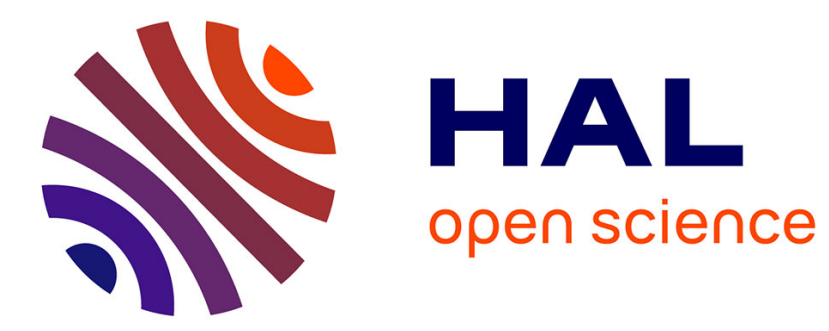

\title{
Le sens du corps. Autour de Richard Shusterman
}

Clotilde Thouret

\section{To cite this version:}

Clotilde Thouret. Le sens du corps. Autour de Richard Shusterman. Critique, 2010, Du Style!, 752-753, pp.179-190. hal-02903131

\section{HAL Id: hal-02903131 \\ https://hal.univ-lorraine.fr/hal-02903131}

Submitted on 24 Aug 2020

HAL is a multi-disciplinary open access archive for the deposit and dissemination of scientific research documents, whether they are published or not. The documents may come from teaching and research institutions in France or abroad, or from public or private research centers.
L'archive ouverte pluridisciplinaire HAL, est destinée au dépôt et à la diffusion de documents scientifiques de niveau recherche, publiés ou non, émanant des établissements d'enseignement et de recherche français ou étrangers, des laboratoires publics ou privés. 


\section{Le sens du corps. Autour de Richard Shusterman}

Richard Shusterman, Sous l'interprétation, trad. Jean-Pierre Cometti, Combas, Éditions de l'éclat, coll. « Tiré à part », 1994, 108p.

Y a-t-il une vie hors de l'interprétation ? En renvoyant toute perception à une situation et à une subjectivité, la pensée de notre post-modernité a répondu par la négative. Cependant, Richard Shusterman propose d'échapper au processus infini qui veut que toute interprétation soit toujours déjà l'interprétation d'une interprétation : pour résister à ce tourbillon de textes qui s'ajoutent sans cesse à d'autres textes, il faut descendre sous l'interprétation, c'est-à-dire être attentif à l'expérience du corps et faire place, de nouveau, à sa manière de faire sens. Cette idée avait déjà vu le jour dans L'art à l'état vif où elle trouvait une illustration évidente dans les analyses fort convaincantes que l'auteur consacrait au rap ${ }^{1}$. Écrit pour les seuls lecteurs francophones à la suite du succès rencontré par ce dernier livre, Sous l'interprétation poursuit ce projet particulièrement joyeux dans sa volonté affichée de retrouver et de cultiver la dimension somatique de notre rapport au monde, et de légitimer par là des arts populaires et les pratiques culturelles qui les accompagnent - même si les expériences esthétiques concrètes se trouvent cette fois étonnamment absents du propos. Puisqu'il s'agit de redonner un corps au destinataire de l'œuvre, le lecteur pourrait en effet s'attendre à ce que des exemples viennent justement donner l'exemple, même sur un mode qui ne soit pas celui de la leçon.

Car son entreprise est, on le voit, indissociablement analytique, pragmatique et pratique : reconnaître l'expérience immédiate et non discursive revient à « prendre la défense de l'ordinaire, tout en recherchant son amélioration » (p. 17). La visée d'un tel pragmatisme

\footnotetext{
$1 \quad$ L'art à l'état vif. La pensée pragmatiste et l'esthétique populaire, trad. Christine Noille, Paris, Éditions de Minuit, 1991. Le débat suscité par la parution de l'ouvrage avait alors trouvé un écho dans cette revue : voir Rainer Rochlitz, «Esthétiques hédonistes », Critique, n 540, mai 1992, p. 353-373. Je voudrais remercier Lise Wajeman : la réflexion exposée ici lui doit beaucoup.
} 
est « d'améliorer la qualité de l'expérience immédiate » et, à cette fin, de « l'élever, et avec elle ses causes somatiques, à une plus grande conscience, voire au langage comme tel» (p. 18). Reconnaître la place d'une compréhension sous l'interprétation et donner ainsi une valeur au versant non linguistique et corporel de la vie désigne implicitement un art de vivre, qui s'attacherait justement à cultiver ce versant trop souvent négligé, en aiguisant la conscience réflexive du corps afin d'améliorer nos perceptions et les usages que nous en avons. Et le philosophe lui-même ne pourra se contenter de la théorie et des mots comme il le fait trop souvent : la philosophie doit se replacer dans la lignée de Socrate, des stoïciens et après eux de Foucault pour se faire discipline de vie incarnée, recherche de la vérité et de la sagesse «à travers une expérience et une exploration somatiques » (p. 104). Son livre plus récent, Conscience du corps. Pour une soma-esthétique, qui s'attache à redonner au corps vivant son rôle de « noyau organisateur de l'expérience », de « site d'appréciation sensorielle et de façonnement créateur de soi», est de ce point de vue l'aboutissement de Sous l'interprétation - mais Shusterman y abandonne la part strictement esthétique de sa réflexion et l'art passe au second plan du propos.

Après le tournant épistémologique, qui marque l'entrée dans la période moderne, et après le tournant linguistique, la pratique philosophique a effectué un tournant herméneutique sous l'impulsion conjointe de la critique littéraire, de l'anthropologie culturelle, de la philosophie du droit, de l'historiographie et du féminisme ${ }^{2}$. Provoquant ce dernier tournant, la mise en question du savoir et du sujet a conféré au concept d'interprétation une importance voire une omniprésence qui en fait le cœur des controverses de la post-modernité et «peutêtre le concept philosophique crucial de notre temps » (p. 7). Une telle accélération sur une route sinueuse n'est pas sans provoquer une sensation de vertige, ou du moins sans susciter la

2 The Interpretive Turn. Philosophy, Science, Culture, éd. David R. Hiley, James F. Bohman, et Richard Shusterman, Ithaca et Londres, Cornell University Press, 1991. 
crainte de perdre quelque chose en chemin. C'est sans doute aussi le sentiment de Richard Shusterman, qui s'attache à préserver une expérience du corps hors de l'interprétation et à réaffirmer la possibilité d'un rapport au monde et aux autres qui soit soustrait à son champ. Il propose de retrouver un terrain stable, quelque chose comme une matière à interpréter, sans toutefois retomber naïvement dans le piège de l'ontologie ou de l'illusion d'une saisie des choses « telles qu'elles sont réellement, de façon univoque, exhaustive et absolue » (p. 60).

Car pour Richard Shusterman, la cause est entendue : rechercher une fondation en vérité des savoirs par une ontologie ou un objectivisme est une impasse et une erreur de la philosophie ; elle y perd une partie de son temps et de son énergie. Sur ce point, l'œuvre de la déconstruction est imparable: la rupture avec le fondationnalisme, qu'il soit humaniste, rationaliste ou analytique, constitue un point de départ déterminant de sa réflexion. Ce qui n'a rien d'évident a priori puisque ses premiers travaux, et notamment sa thèse, s'inscrivaient dans le droit fil de la philosophie analytique d'Oxford. Si, et il y insiste lui-même, son approche était alors déjà profondément pluraliste, il n'a amorcé un tournant pragmatiste qu'après avoir rencontré la théorie post-structuraliste et la critique déconstructionniste, et en travaillant plus avant les textes de James et Dewey. Sans pour autant que sa position s'identifie à celle de Rorty, bien au contraire : d'abord parce qu'il engage un dialogue explicite avec la French theory, et surtout parce qu'il refuse un relativisme généralisé et un textualisme exacerbé. Quand le pragmatisme rortien veut substituer la notion de langage à celle d'expérience, Shusterman fait valoir l'existence et l'importance d'une expérience immédiate et non-discursive. En outre, il fait sien le projet de Dewey qui, en libérant « la philosophie de sa quête des fondements, voulait mettre son entreprise au service de réformes concrètes » (p. 80); «au lieu de se vouloir une méta-science, [...] [la philosophie] doit être une forme de critique culturelle visant à redécrire le monde dont nous avons l'expérience, et à reconstruire nos institutions et nos pratiques de manière à améliorer la qualité de nos vies » 
(p. 81). Son pragmatisme est donc aussi un méliorisme : la réflexion philosophique ne se dissocie pas de la revendication d'un engagement éthique.

Le projet de Sous l'interprétation s'inscrit dans cette perspective: contre les « distorsions appauvrissantes » d'une philosophie issue du tournant linguistique (celle de Derrida ou de Rorty) qui « subordonne toute expérience humaine au langage ou à un texte, de la même façon qu'elle assimile la compréhension à l'interprétation » (p. 9), il s'agit de reconnaître une dimension de l'expérience qui ne relève pas toujours déjà de l'interprétation et qui pourtant « fait sens ». Circonscrire ainsi la place d'un sens qui échappe au langage articulé parce qu'il est produit par une réponse somatique non discursive permettra de redonner un espace dans le champ philosophique à une dimension non linguistique, et pourtant valable, de l'expérience et donc de la légitimer.

On perçoit d'emblée le rôle stratégique de l'art et du corps dans une telle entreprise de pensée : l'œuvre d'art est le lieu par excellence de la signification et l'objet privilégié de l'herméneutique ; mais elle est aussi à l'origine d'une expérience somatique qui se soustrait $a$ priori à la pensée réflexive et au langage : les émotions suscitées par une histoire tragique, ou bien encore la danse, même minimale, provoquée par un morceau de musique. Pour faire sens l'art n'exige pas toujours de réponse verbale : cela vaut pour le danseur ou le musicien qui interprètent une œuvre, tout autant que pour le spectateur ou l'auditeur qui à leur tour donnent un sens à l'œuvre qu'ils regardent ou écoutent, par cette simple réaction du corps, directe, immédiate et irréfléchie, sans nécessairement tenter de décrire ou de reconstruire le sens de l'œuvre ${ }^{3}$.

La pensée et l'argumentation du philosophe progressent d'une manière que l'on

\footnotetext{
3 Dès The Object of Literary Criticism, tiré de sa thèse et publié en 1984, Shusterman identifiait plusieurs logiques et plusieurs pratiques interprétatives, toutes ne visant pas la vérité ou la connaissance : il proposait ainsi de concevoir l'interprétation, non pas comme un seul jeu interprétatif (au sens où Wittgenstein parle de «jeu de langage »), mais comme une famille de jeux. Ce pluralisme est sans doute encore accusé dans la publication française de l'ouvrage pour laquelle il a justement réécrit le chapitre intitulé «La logique de l'interprétation » (L'Objet de la critique littéraire, trad. Nicolas Vieillescazes, Questions théoriques, 2009).
} 
pourrait qualifier de «spatiale». Le pragmatisme trace une voie moyenne, un chemin entre philosophie analytique et déconstruction. Considérant et réfutant les arguments, abandonnant et conservant les idées des uns comme des autres, Shusterman dessine les frontières de cette expérience immédiate qui s'identifie à une compréhension somatique. Il procède en trois temps. À la faveur de la question de l'unité organique de l'objet d'art, point de clivage entre les philosophes analytiques et les déconstructionnistes, le plaisir esthétique est amené à jouer un rôle central dans la définition de l'œuvre d'art. Le deuxième chapitre écarte d'une part l'universalisme herméneutique et d'autre part la position fondationnaliste pour ménager la place d'une compréhension qui ne soit ni de l'ordre d'une herméneutique ni de nature fondationnelle, et qui ne s'identifie ni à un sens élaboré dans le discours ni à un accès objectif à une œuvre qui resterait pour toujours identique à elle-même. Enfin, la notion d'« expérience immédiate » et non discursive reprise à Dewey est détachée de son éventuelle fonction fondationnelle pour acquérir un rôle esthétique au sens premier du terme, celui de l'articulation du corps et de l'esprit; elle est ainsi constituée en fin pratique et en outil opportun dans nos rapports avec le monde.

Prendre pour objet de la réflexion le concept d'unité organique et plus particulièrement d'unité organique de l'œuvre d'art n'a pas seulement pour visée de démontrer la nécessité d'une voie moyenne pragmatiste entre les apories de la philosophie analytique et de la déconstruction. C'est aussi entrer de plain pied dans le problème de l'interprétation puisque celui-ci s'articule à la question de l'unité organique : dire que l'œuvre d'art possède une unité organique revient à affirmer qu'elle a une signification objective, tandis que considérer l'œuvre comme soumise à un principe de fragmentation ou de différentiation réfute l'idée d'une signification objective que l'interprétation pourrait reconstruire.

Ici comme ailleurs, pour Shusterman, la philosophie analytique comme la déconstruction vont trop loin. Face à un tableau, comme à un corps vivant ou à une salade 
(p. 26), il est difficile de ne pas reconnaître le bien fondé du principe organiciste : le tout possède une valeur intrinsèque qui n'est pas réductible à la somme des parties constituantes, même si cette totalité n'existe pas indépendamment des parties. Mais il n'est pas nécessaire d'affirmer que les objets, les parties et les totalités sont des réalités fixes et autonomes, toujours identiques à elles-mêmes. En particulier pour l'art, la temporalité et les pratiques entrent en jeu : il n'est que de considérer les ready-made d'un Duchamp. Parallèlement, la critique déconstructionniste de l'unité esthétique doit être entendue: la multiplicité des lectures et des interprétations plaide en sa faveur; par ailleurs, comme le montre Jonathan Culler, la distinction entre un intérieur de l'œuvre et un extérieur est très problématique (par exemple, le cadre d'une œuvre picturale fait aussi partie de ce qui la constitue). Cependant, outre que la logique de la différance reconduit l'idée d'un tout qui détermine le sens de chaque partie, l'idée d'unité doit être récupérée, non pas dans une perspective ontologique mais pour des raisons pratiques. Certes, l'unité d'un texte et, partant, sa signification, ne sont pas de l'ordre d'une essence parce qu'ils dépendent toujours d'un contexte et d'une perspective herméneutique, mais postuler cette unité se justifie comme "une stratégie de lecture et un critère interprétatif de pertinence et de centralité » : le plaisir esthétique naît souvent de ce sentiment d'unité qui contraste avec la fragmentation et l'incomplétude de l'expérience; en outre, l'unité est l'horizon de la démarche interprétative, la condition nécessaire de l'intelligibilité. Shusterman déplace donc la réflexion de la question finalement futile - de l'essence vers celle des pratiques et recentre ainsi l'argumentation sur le destinataire de l'œuvre, sur ses habitudes et les fins qu'il poursuit.

Le deuxième chapitre constitue le cœur de l'ouvrage : il élabore le concept de compréhension, distinct à la fois de l'interprétation et de l'objectivité qui donnerait un accès à une vérité absolue et univoque. Contre l'universalisme herméneutique de tous les antifondationnalistes, il s'agit de montrer que « nos échanges intelligents et sensés avec le monde 
incluent une expérience, une activité et une compréhension non interprétationnelles » (p. 53). Même si le sens est toujours situé, même si la perception d'une œuvre d'art est indissociable d'une perspective, l'interprétation ne subsume pas toute l'expérience. Shusterman renvoie dos à dos les tenants du tout-interprétation (pragmatistes radicaux, comme Stanley Fish, et déconstructionnistes) et les tenants du fondationnalisme parce qu'ils partagent un même présupposé erroné, à savoir : ce qui n'est pas de nature fondationnelle repose forcément sur une interprétation. En répondant aux fondationnalistes sur le terrain ontologique, les partisans de l'universalisme herméneutique affirment qu'une compréhension non interprétée est impossible, car elle devrait être conçue en termes fondationnels. Ils restent en quelque sorte prisonniers de la polémique et des termes dans lesquels elle est posée, et oublient ainsi tout un pan du réel et de l'expérience. Pour fonder logiquement la possibilité d'une compréhension non interprétative, Shusterman peut récupérer des idées des deux camps opposés parce qu'il se débarrasse de leurs thèses «radicales » : il reprend aux fondationnalistes un niveau sous l'interprétation tout en mettant à l'écart l'idée que l'on puisse saisir les choses telles qu'elles sont réellement, de façon univoque, exhaustive et absolue; il reprend aux antifondationnalistes leur pluralisme, leur refus de la croyance en une essence ou une vérité intemporelle des objets, tout en mettant à l'écart l'idée que toute expérience est toujours déjà linguistique et interprétative. Le double mouvement de pensée apparaît avec évidence dans une affirmation comme celle-ci : «Ainsi, il n'est absolument pas nécessaire de nier que la compréhension vraie peut elle-même être liée à des perspectives partiales et plurielles, ni d'affirmer que toute compréhension devant être liée à une perspective, il doit nécessairement y avoir une interprétation » (p. 60).

Une fois circonscrite négativement, la compréhension peut être définie positivement. Le corps est alors appelé à jouer un rôle décisif. À l'interprétation, qui relève d'une opération langagière, consciente et délibérée, Shusterman oppose l'exemple de la descente d'un escalier 
ou de l'exactitude du mouvement du danseur : ces deux expériences présupposent bien une compréhension sélective (où mettre les pieds, où arrêter un mouvement), mais elle n'est ni consciente ni délibérée, elle demeure dans l'ordre de l'immédiateté et de la réaction corporelle. « Structuration active de ce qui est rencontré », la compréhension somatique n'est pas un simple «reflet passif», mais elle ne relève pas pour autant d'une expérience linguistique : aucun décodage des signes n'intervient ici et elle n'exige aucune articulation dans le langage. On voit de nouveau comment le philosophe utilise un camp contre l'autre pour dessiner la troisième voie pragmatique : il joue Gadamer contre Wittgenstein, puis l'inverse.

La nature de la distinction entre compréhension et interprétation se trouve ensuite précisée. Elle est avant tout fonctionnelle et relationnelle, et non ontologique. Elle joue d'abord un rôle logique: si tout rapport au monde, si toute réception d'une œuvre est interprétation, comment la définir? La compréhension donne quelque chose à quoi l'opposer et lui donne son sens. En outre, elle ce qui assoit et guide l'interprétation : en effet, «il n'existe pas de dichotomie rigide et absolue, mais une continuité essentielle et des degrés d'interdépendance entre la compréhension et l'interprétation » (p. 71). Enfin, elle ouvre sur une défense de l'ordinaire, elle permet de valoriser une compréhension dénuée d'efforts, irréfléchie, et de contrer l'intellectualisme qui menace souvent les théoriciens de l'universalisme herméneutique, en particulier lorsqu'il est question de la réception des œuvres et donc de la distinction entre art et non-art.

Les détours qu'emprunte l'ouvrage par les débats philosophiques plus ou moins contemporains pourront sembler un peu longs, et la réfutation des arguments des uns et des autres parfois superflue au regard de la proposition du philosophe. Mais, outre qu'ils sont sans doute nécessaires pour faire la place du pragmatisme dans le champ particulièrement riche et 
vivant de la philosophie américaine, cette proposition a quelque chose d'« engageant»l'anglais dirait compelling - voire de réjouissant, notamment pour celui qui s'intéresse, éventuellement de manière non philosophique, aux pratiques artistiques plus anciennes et qui croit qu'elles peuvent concerner notre présent. Car, finalement, pour sortir des impasses de la modernité et contrer les tendances d'une post-modernité qui semble annoncer la fin de l'art, Shusterman suggère de redonner un corps au destinataire de l'œuvre, dans l'expérience comme dans l'analyse. Si, après L'art à l'état vif, il continue de souligner la matérialité du corps du lecteur, de l'auditeur et du spectateur, c'est d'une part pour qu'il existe de nouveau pour le critique et le philosophe de l'art, et d'autre part pour augmenter la conscience réflexive que peut en avoir ce lecteur, auditeur ou spectateur. En effet, ce corps, qui est au cœur de l'expérience esthétique parce qu'il est le lieu de l'aisthêsis et de l'interaction de du sujet avec le monde, est souvent ignoré par toute une tradition esthétique qui affirme l'absence de finalité de l'art et qui identifie toute réception à une interprétation. Redonner un corps au destinataire peut aussi s'entendre en un sens figuré car tenir compte de l'effet des œuvres sur les corps revient à placer un destinataire face à l'œuvre, à renouer le lien entre l'œuvre et ses destinataires - même si leurs identités et leurs réactions demeurent indéterminées et plurielles -, ce qui implique une finalité pratique voire éthique de l'art.

Une telle réflexion fait ainsi irrésistiblement écho aux pensées de l'art d'avant la rupture esthétique : il n'est que de considérer les traités sur la peinture ou les poétiques des $\mathrm{XVI}^{\mathrm{e}}$ et des $\mathrm{XVII}^{\mathrm{e}}$ siècles pour voir l'attention que portent les théoriciens et les artistes aux passions du lecteur ou du spectateur, notamment afin d'inscrire dans une perspective morale l'effet rhétorique de l'œuvre ${ }^{4}$. On peut alors trouver surprenant voire paradoxal que Shusterman ne renvoie pas aux cadres esthétiques du début de l'époque moderne, dont il

\footnotetext{
$4 \quad$ Voir notamment François Lecercle, «De honestate imaginum. L’honnête dans les controverses sur l'image au XVI siècle », dans La Catégorie de l'honneste dans la culture du XVI siècle, Saint-Etienne, Presses de l'université de Saint-Etienne, 1985, p. 107-121.
} 
retrouve certains aspects par son parti-pris pragmatiste ${ }^{5}$. Sa défense de l'ordinaire, il est vrai, justifie son intérêt pour des arts ou des pratiques culturelles comme le rap ou la techno. Par ailleurs, il aurait pris le risque de voir ses thèses infléchies vers une idée que la pensée postmoderne associe parfois au seuil de la modernité et au «classicisme» en particulier, et à laquelle elle s'oppose, celle d'un destinataire connu de l'auteur et à qui il s'adresse, programmant sa réception et sa réaction sensible. Mais, outre que cette association est erronée, les arts des siècles renaissants et « classiques » ménageant justement la multiplicité des publics et l'imprévisibilité des réceptions ${ }^{6}$, on regrettera que le philosophe n'y puise pas, éventuellement pour les discuter, des arguments et des exemples, qui donneraient plus de chair aux réflexions paradoxalement abstraites de Sous l'interprétation. Le théâtre pourrait légitimement retenir son attention : les dramaturges, les théoriciens du théâtre et même, dans leurs dénonciations des complaisances que les œuvres ont pour le corps, les plus fervents adversaires de cet art, proposent des réflexions extrêmement élaborées sur la manière dont la compréhension du corps, à travers les émotions qui le traversent lors de la représentation, s'articule à l'interprétation. Les reformulations de la catharsis aristotélicienne tendent par exemple à faire de la réaction passionnelle, et donc somatique, du spectateur une compréhension de l'œuvre. Ainsi Corneille, dans Le Discours de la tragédie, analyse-t-il le jeu des émotions tragiques, «la pitié d'un malheur où nous voyons tomber nos semblables » qui «nous porte à la crainte d'un pareil pour nous», comme un double processus d'identification et de dissociation entre le spectateur et le personnage. Ce double processus donne son sens à l'œuvre puisque c'est à partir de lui que le spectateur est amené à identifier la cause de ce malheur qu'il désire éviter, c'est-à-dire « cette passion qui plonge à nos yeux

\footnotetext{
$5 \quad$ Il est vrai que La fin de l'expérience esthétique suggère, pour sortir de la crise de la modernité, de se tourner «vers des expériences esthétiques antérieures ou extérieures à la naissance présumée de l'art dans la modernité occidentale » (La fin de l'expérience esthétique, trad. Jean-Pierre Cometti, Fabienne Gaspari et Anne Combarnous, Publications de l'Université de Pau, 1999).

6 Voir Hélène Merlin-Kajman, « Un siècle classico-baroque ? », XVIIe siècle, 2004/2, n²23, p. 163-172.
} 
dans ce malheur les personnes que nous plaignons ${ }^{7} »$. L'expérience somatique, immédiate et irréfléchie, de la représentation tragique fait sens avec l'œuvre, hors de toute articulation langagière, tout en conduisant vers l'interprétation : elle gouverne la lecture de l'œuvre et lui confère en outre sa fonction pratique puisqu'elle porte le spectateur à l'action vertueuse.

Mais l'art dramatique n'est pas seul à susciter chez son spectateur une expérience somatique qui l'engage à améliorer son rapport avec le monde ; pour asseoir ses réflexions théoriques et former un pendant plus « institutionnel » au rap ou au rock, le cinéma aurait pu fournir à Shusterman une autre matière, sans doute plus en accord avec sa célébration du corps que la tragédie du XVII ${ }^{\mathrm{e}}$ siècle, difficilement dissociable de la condamnation des passions. Je profiterai de cette place qu'il a laissée vide pour évoquer la dernière œuvre d'Alain Guiraudie. Comme ses autres films mais peut-être plus encore, Le roi de l'évasion s'appuie sur « un retour allègre et impétueux de la dimension somatique ${ }^{8}$ » et donne lieu à une expérience immédiate et non-discursive, qui, par son effet pragmatique évident, suscite la bonne disposition à l'égard d'autrui par une générosité de regard. Le film fonctionne comme un exercice corporel, au sens où Ignace de Loyola parlait d'exercices spirituels : il propose au spectateur d'exercer son regard sur le monde pour en faire un regard généreux et désirant. Cela passe d'abord par la joie devant le plaisir et l'exercice des corps des personnages, exaltés par la caméra, et dans la fiction, par le désir et une drogue naturelle du nom de « dourougne ». Cette joie naît de la course, de voir courir, longuement, Armand et Curly : dans la forêt ensoleillée quand le vendeur de matériel agricole et la jeune fille essaient d'échapper aux parents et à la police pour s'aimer librement, mais aussi à travers champs quand le premier veut s'évader du couple que Curly aimerait construire avec lui. Elle naît aussi du plaisir de la

\footnotetext{
$7 \quad$ Pierre Corneille, Discours de la tragédie, dans Euvres complètes, t. 3, éd. Georges Couton, Paris, Gallimard, «Bibliothèque de la Pléiade », 1987, p. 143. Sur la place des passions dans la réflexion théorique sur le théâtre au XVII ${ }^{\mathrm{e}}$ siècle et dans celle de Corneille en particulier, voir John D. Lyons, Kingdom of Disorder. The Theory of Tragedy in Classical France, Purdue University Press, 1999, Chap. 2 « Passion in the Age of Reason », p. 43-82 ; H. Merlin-Kajman, L'absolutisme dans les lettres et la théorie des deux corps. Passions et politique, Paris, Champion, 2000, p. 21-101.

Ce sont les mots que Shusterman emploie au sujet du rock dans L'art à l'état vif, éd. cit., p. 159.
} 
sexualité des autres car le film de Guiraudie élabore pour le spectateur un plaisir entièrement tourné vers l'autre, ce que l'art cinématographique ne parvient que très rarement à faire lorsqu'il représente l'acte sexuel. Ce plaisir n'a rien de narcissique parce que sa représentation n'a recourt à aucun des topoi de l'érotique convenue du cinéma; elle est surprenante parce que justement les gens sont ordinaires : ce sont des corps amoureux que l'on ne voit guère au cinéma, des corps d'ouvriers et de paysans, d'hommes mûrs, parfois gros, mais d'une beauté évidente. Cette représentation ne reconduit aucune norme ni aucun rapport de domination, et montre une sexualité à la fois intense et apaisée, même si elle l'objet de questionnements. Le film suscite ainsi chez le spectateur une réjouissance sensible, qui se passe de toute articulation dans le langage. De la même manière qu'Armand fonctionne dans la fiction comme un principe actif de désir puisque tous les personnages qui le rencontrent le désirent intensément, l'œuvre filmique précipite le désir chez son spectateur. Par la fréquentation de ces images, il gagne un corps plus léger et plus libre, il augmente sa capacité de sentir et de voir, et de le faire généreusement.

Clotilde Thouret 